\title{
A Probabilistic Approach to Problems Parameterized Above or Below Tight Bounds*
}

\author{
Gregory Gutin ${ }^{\dagger} \quad$ Eun Jung Kim ${ }^{\ddagger} \quad$ Stefan Szeider ${ }^{\S}$ \\ Anders Yeo
}

\begin{abstract}
We introduce a new approach for establishing fixed-parameter tractability of problems parameterized above tight lower bounds or below tight upper bounds. To illustrate the approach we consider three problems of this type of unknown complexity that were introduced by Mahajan, Raman and Sikdar (J. Comput. Syst. Sci. 75, 2009). We show that a generalization of one of the problems and non-trivial special cases of the other two are fixed-parameter tractable.
\end{abstract}

\section{Introduction}

A parameterized problem $\Pi$ can be considered as a set of pairs $(I, k)$ where $I$ is the main part and $k$ (usually an integer) is the parameter. $\Pi$ is called fixedparameter tractable (FPT) if membership of $(I, k)$ in $\Pi$ can be decided in time $O\left(f(k)|I|^{c}\right)$, where $|I|$ denotes the size of $I, f(k)$ is a computable function, and $c$ is a constant independent of $k$ and $I$ (for further background and terminology on parameterized complexity we refer the reader to the monographs [6, 8, 17]). If the nonparameterized version of $\Pi$ (where $k$ is just a part of the input) is NP-hard, then the function $f(k)$ must be superpolynomial provided $\mathrm{P} \neq \mathrm{NP}$. Often $f(k)$ is "moderately exponential," which makes the problem practically feasible for small values of $k$. Thus, it is important to parameterize a problem in such a way that the instances with small values of $k$ are of real interest.

Consider the following well-known problem: given a digraph $D=(V, A)$, find an acyclic subdigraph of $D$ with the maximum number of arcs. We can

\footnotetext{
*An extended abstract of this paper will appear in the Proceedings of IWPEC 2009.

$\dagger$ Department of Computer Science, Royal Holloway University of London, Egham, Surrey TW20 0EX, England, UK, gutin@cs.rhul.ac.uk

${ }^{\ddagger}$ Department of Computer Science, Royal Holloway University of London, Egham, Surrey TW20 0EX, England, UK, eunjung@cs.rhul.ac.uk

$\S$ Department of Computer Science, Durham University, Durham DH1 3LE, England, UK, stefan@szeider.net

I Department of Computer Science, Royal Holloway University of London, Egham, Surrey TW20 0EX, England, UK, anders@cs.rhul.ac.uk
} 
parameterize this problem "naturally" by asking whether $D$ contains an acyclic subdigraph with at least $k$ arcs. It is easy to prove that this parameterized problem is fixed-parameter tractable by observing that $D$ always has an acyclic subdigraph with at least $|A| / 2$ arcs. (Indeed, consider a bijection $\alpha: V \rightarrow$ $\{1, \ldots,|V|\}$ and the following subdigraphs of $D:(V,\{x y \in A: \alpha(x)<\alpha(y)\})$ and $(V,\{x y \in A: \alpha(x)>\alpha(y)\})$. Both subdigraphs are acyclic and at least one of them has at least $|A| / 2$ arcs.) However, $k \leq|A| / 2$ for every small value of $k$ and almost every practical value of $|A|$ and, thus, our "natural" parameterization is of almost no practical or theoretical interest.

Instead, one should consider the following parameterized problem: decide whether $D=(V, A)$ contains an acyclic subdigraph with at least $|A| / 2+k$ arcs. We choose $|A| / 2+k$ because $|A| / 2$ is a tight lower bound on the size of a largest acyclic subdigraph. Indeed, the size of a largest acyclic subdigraph of a symmetric digraph $D=(V, A)$ is precisely $|A| / 2$. (A digraph $D=(V, A)$ is symmetric if $x y \in A$ implies $y x \in A$.)

In a recent paper [16] Mahajan, Raman and Sikdar provided several examples of problems of this type and argued that a natural parameterization is one above a tight lower bound for maximization problems, and below a tight upper bound for minimization problems. Furthermore, they observed that only a few non-trivial results are known for problems parameterized above a tight lower bound [11, 12, 14, 15, and they listed several problems parameterized above a tight lower bound whose complexity is unknown. The difficulty in showing whether such a problem is fixed-parameter tractable can be illustrated by the fact that often we even do not know whether the problem is in XP, i.e., can be solved in time $O\left(|I|^{g(k)}\right)$ for a computable function $g(k)$. For example, it is non-trivial to see that the above-mentioned digraph problem is in XP when parameterized above the $|A| / 2$ bound.

In this paper we introduce the Strictly Above/Below Expectation Method ( $S A B E M)$, a novel approach for establishing the fixed-parameter tractability of maximization problems parameterized above tight lower bounds and minimization problems parameterized below tight upper bounds. The new method is based on probabilistic arguments and utilizes certain probabilistic inequalities. We will state the equalities in the next section, and in the subsequent sections we will apply SABEM to three open problems posed in 16 .

Now we give a very brief description of the new method with respect to a given problem $\Pi$ parameterized above a tight lower bound or below a tight upper bound. We first apply some reductions rules to reduce $\Pi$ to its special case $\Pi^{\prime}$. Then we introduce a random variable $X$ such that the answer to $\Pi$ is YES if and only if $X$ takes, with positive probability, a value greater or equal to the parameter $k$. Now using some probabilistic inequalities on $X$, we derive upper bounds on the size of No-instances of $\Pi^{\prime}$ in terms of a function of the parameter $k$. If the size of a given instance exceeds this bound, then we know the answer is YES; otherwise, we produce a problem kernel [6]. In many cases, we obtain problem kernels of polynomial size.

In Section 3, we consider the LINEAR ORDERING problem, a generalization of the problem discussed above: Given a digraph $D=(V, A)$ in which each arc $i j$ 
has a positive integral weight $w_{i j}$, find an acyclic subdigraph of $D$ of maximum weight. Observe that $W / 2$, where $W$ is the sum of all arc weights, is a tight lower bound for LinEAR ORDERING. We prove that the problem parameterized above $W / 2$ is fixed-parameter tractable and admits a quadratic kernel. Note that this parameterized problem generalizes the parameterized maximum acyclic subdigraph problem considered in [16]; thus, our result answers the corresponding open question of [16.

In Section 4, we consider the problem Max Lin-2: Given a system of $m$ linear equations $e_{1}, \ldots, e_{m}$ in $n$ variables over $\mathrm{GF}(2)$, and for each equation $e_{j}$ a positive integral weight $w_{j}$; find an assignment of values to the $n$ variables that maximizes the total weight of the satisfied equations. We will see that $W / 2$, where $W=w_{1}+\cdots+w_{m}$, is a tight lower bound for Max Lin-2. The complexity of the problem parameterized above $W / 2$ is open [16]. We prove that the following three special cases of the parameterized problem are fixedparameter tractable: (1) there is a set $U$ of variables such that each equation has an odd number of variables from $U,(2)$ there is a constant $r$ such that each equation involves at most $r$ variables, (3) there is a constant $\rho$ such that any variable appears in at most $\rho$ equations. For all three cases we obtain kernels with $O\left(k^{2}\right)$ variables and equations. We also show that if we allow the weights $w_{j}$ to be positive reals, the problem is NP-hard already if $k=1$ and each equation involves two variables.

In Section 5, we consider the problem MAX EXACT $r$-SAT: given an exact $r$-CNF formula $\mathcal{C}$ with $m$ clauses (i.e., a CNF formula where each clause contains exactly $r$ distinct literals), find a truth assignment that satisfies the maximum number of clauses. Here a tight lower bound is $\left(1-2^{-r}\right) m$; the complexity of the problem parameterized above $\left(1-2^{-r}\right) m$ is an open question [16. This seems to be the most difficult problem of the three considered. We obtain a quadratic kernel for a non-trivial special case of this problem.

In Section 6, we briefly mention minimization problems parameterized below tight upper bounds, provide further discussions of problems considered in this paper and point out to a very recent result obtained using our new method.

\section{Probabilistic Inequalities}

In our approach we introduce a random variable $X$ such that the answer to the problem parameterized above a tight lower bound or below a tight upper bound is YES if and only if $X$ takes with positive probability a value greater or equal to the parameter $k$.

In this paper all random variables are real. A random variable is discrete if its distribution function has a finite or countable number of positive increases. A random variable $X$ is a symmetric if $-X$ has the same distribution function as $X$. If $X$ is discrete, then $X$ is symmetric if and only if $\operatorname{Prob}(X=a)=$ $\operatorname{Prob}(X=-a)$ for each real $a$. Let $X$ be a symmetric variable for which the first moment $\mathbb{E}(X)$ exists. Then $\mathbb{E}(X)=\mathbb{E}(-X)=-\mathbb{E}(X)$ and, thus, $\mathbb{E}(X)=0$. The following is easy to prove [18]. 
Lemma 1. If $X$ is a symmetric random variable and $\mathbb{E}\left(X^{2}\right)<\infty$, then

$$
\operatorname{Prob}\left(X \geq \sqrt{\mathbb{E}\left(X^{2}\right)}\right)>0 .
$$

See Sections 3 and 4 for applications of Lemma 1 Unfortunately, often $X$ is not symmetric, but Lemma 2 provides an inequality that can be used in many such cases. This lemma was proved by Alon et al. [1]; a weaker version was obtained by Håstad and Venkatesh [13.

Lemma 2. Let $X$ be a random variable and suppose that its first, second and forth moments satisfy $\mathbb{E}(X)=0, \mathbb{E}\left(X^{2}\right)=\sigma^{2}>0$ and $\mathbb{E}\left(X^{4}\right) \leq b \sigma^{4}$, respectively. Then $\operatorname{Prob}\left(X>\frac{\sigma}{4 \sqrt{b}}\right) \geq \frac{1}{4^{4 / 3} b}$.

Since it is often rather nontrivial to evaluate $\mathbb{E}\left(X^{4}\right)$ in order to check whether $\mathbb{E}\left(X^{4}\right) \leq b \sigma^{4}$ holds, one can sometimes use the following extension of Khinchin's Inequality by Bourgain [4].

Lemma 3. Let $f=f\left(x_{1}, \ldots, x_{n}\right)$ be a polynomial of degree $r$ in $n$ variables $x_{1}, \ldots, x_{n}$ with domain $\{-1,1\}$. Define a random variable $X$ by choosing a vector $\left(\epsilon_{1}, \ldots, \epsilon_{n}\right) \in\{-1,1\}^{n}$ uniformly at random and setting $X=f\left(\epsilon_{1}, \ldots, \epsilon_{n}\right)$. Then, for every $p \geq 2$, there is a constant $c_{p}$ such that

$$
\left(\mathbb{E}\left(|X|^{p}\right)\right)^{1 / p} \leq\left(c_{p}\right)^{r}\left(\mathbb{E}\left(X^{2}\right)\right)^{1 / 2} .
$$

In particular, $c_{4} \leq 2^{3 / 2}$.

\section{Linear Ordering}

Let $D=(V, A)$ be a digraph with no loops or parallel arcs in which every arc $i j$ has a positive weight $w_{i j}$. The problem of finding an acyclic subdigraph of $D$ of maximum weight, known as Linear Ordering, has applications in economics [2]. Let $n=|V|$ and consider a bijection $\alpha: V \rightarrow\{1, \ldots, n\}$. Observe that the subdigraphs $(V,\{i j \in A: \alpha(i)<\alpha(j)\})$ and $(V,\{i j \in A: \alpha(i)>$ $\alpha(j)\})$ are acyclic. Since the two subdigraphs contain all $\operatorname{arcs}$ of $D$, at least one of them has weight at least $W / 2$, where $W=\sum_{i j \in A} w_{i j}$, the weight of $D$. Thus, $W / 2$ is a lower bound on the maximum weight of an acyclic subdigraph of $D$. Consider a digraph $D$ where for every $\operatorname{arc} i j$ of $D$ there is also an $\operatorname{arc} j i$ of the same weight. Each maximum weight subdigraph of $D$ has weight exactly $W / 2$. Hence the lower bound $W / 2$ is tight.

\section{Linear Ordering Above Tight Lower Bound (LOALB)}

Instance: A digraph $D=(V, A)$, each arc $i j$ has an integral positive weight $w_{i j}$, and a positive integer $k$.

Parameter: The integer $k$.

Question: Is there an acyclic subdigraph of $D$ of weight at least $W / 2+k$, where $W=\sum_{i j \in A} w_{i j}$ ? 
Mahajan, Raman, and Sikdar [16] asked whether LOALB is fixed-parameter tractable for the special case when all arcs are of weight 1 (i.e., $D$ is unweighted). In this section we will prove that LOALB admits a kernel with $O\left(k^{2}\right)$ arcs; consequently the problem is fixed-parameter tractable. Note that if we allow weights to be positive reals, then we can show, similarly to the NP-completeness proof given in the next section, that LOALB is NP-complete already for $k=1$.

Consider the following reduction rule:

Reduction Rule 1. Assume $D$ has a directed 2-cycle iji; if $w_{i j}=w_{j i}$ delete the cycle, if $w_{i j}>w_{j i}$ delete the arc ji and replace $w_{i j}$ by $w_{i j}-w_{j i}$, and if $w_{j i}>w_{i j}$ delete the arc ij and replace $w_{j i}$ by $w_{j i}-w_{i j}$.

It is easy to check that the answer to LOALB for a digraph $D$ is YES if and only if the answer to LOALB is YES for a digraph obtained from $D$ using the reduction rule as long as possible.

Let $D=(V, A)$ be an oriented graph, let $n=|V|$ and $W=\sum_{i j \in A} w_{i j}$. Consider a random bijection: $\alpha: V \rightarrow\{1, \ldots, n\}$ and a random variable $X(\alpha)=$ $\frac{1}{2} \sum_{i j \in A} \epsilon_{i j}(\alpha)$, where $\epsilon_{i j}(\alpha)=w_{i j}$ if $\alpha(i)<\alpha(j)$ and $\epsilon_{i j}(\alpha)=-w_{i j}$, otherwise. It is easy to see that $X(\alpha)=\sum\left\{w_{i j}: i j \in A, \alpha(i)<\alpha(j)\right\}-W / 2$. Thus, the answer to LOALB is YES if and only if there is a bijection $\alpha: V \rightarrow\{1, \ldots, n\}$ such that $X(\alpha) \geq k$. Since $\mathbb{E}\left(\epsilon_{i j}\right)=0$, we have $\mathbb{E}(X)=0$.

Let $W^{(2)}=\sum_{i j \in A} w_{i j}^{2}$. We will prove the following:

Lemma 4. $\mathbb{E}\left(X^{2}\right) \geq W^{(2)} / 12$.

Proof. Let $\mathrm{N}^{+}(i)$ and $\mathrm{N}^{-}(i)$ denote the sets of out-neighbors and in-neighbors of a vertex $i$ in $D$. By the definition of $X$,

$$
4 \cdot \mathbb{E}\left(X^{2}\right)=\sum_{i j \in A} \mathbb{E}\left(\epsilon_{i j}^{2}\right)+\sum_{i j, p q \in A} \mathbb{E}\left(\epsilon_{i j} \epsilon_{p q}\right),
$$

where the second sum is taken over ordered pairs of distinct arcs. Clearly, $\sum_{i j \in A} \mathbb{E}\left(\epsilon_{i j}^{2}\right)=W^{(2)}$. To compute $\sum_{i j, p q \in A} \mathbb{E}\left(\epsilon_{i j} \epsilon_{p q}\right)$ we consider the following cases:

Case 1: $\{i, j\} \cap\{p, q\}=\emptyset$. Then $\epsilon_{i j}$ and $\epsilon_{p q}$ are independent and $\mathbb{E}\left(\epsilon_{i j} \epsilon_{p q}\right)=$ $\mathbb{E}\left(\epsilon_{i j}\right) \mathbb{E}\left(\epsilon_{p q}\right)=0$.

Case 2a: $|\{i, j\} \cap\{p, q\}|=1$ and $i=p$. Since the probability that $i<$ $\min \{j, q\}$ or $i>\max \{j, q\}$ is $2 / 3, \epsilon_{i j} \epsilon_{i q}=w_{i j} w_{i q}$ with probability $\frac{2}{3}$ and $\epsilon_{i j} \epsilon_{i q}=$ $-w_{i j} w_{i q}$ with probability $\frac{1}{3}$. Thus, for every $i \in V$ we have $\sum_{i j, i q \in A} \mathbb{E}\left(\epsilon_{i j} \epsilon_{i q}\right)=$ $\frac{1}{3} \sum\left\{w_{i j} w_{i q}: j \neq q \in N^{+}(i)\right\}=\frac{1}{3}\left(\sum_{j \in N^{+}(i)} w_{i j}\right)^{2}-\frac{1}{3} \sum_{j \in N^{+}(i)} w_{i j}^{2}$.

Case 2b: $|\{i, j\} \cap\{p, q\}|=1$ and $j=q$. Similarly to Case 2a, we obtain $\sum_{i j, p j \in A} \mathbb{E}\left(\epsilon_{i j} \epsilon_{p j}\right)=\frac{1}{3}\left(\sum_{i \in N^{-}(j)} w_{i j}\right)^{2}-\frac{1}{3} \sum_{i \in N^{-}(j)} w_{i j}^{2}$. 
Case 3a: $\quad|\{i, j\} \cap\{p, q\}|=1$ and $i=q$. Since $\epsilon_{i j} \epsilon_{p i}=w_{i j} w_{p i}$ with probability $\frac{1}{3}$ and $\epsilon_{i j} \epsilon_{p i}=-w_{i j} w_{p i}$ with probability $\frac{2}{3}$, we obtain $\sum_{i j, p i \in A} \mathbb{E}\left(\epsilon_{i j} \epsilon_{p i}\right)=$ $-\frac{1}{3} \sum\left\{w_{i j} w_{p i}: j \in N^{+}(i), p \in N^{-}(i)\right\}=-\frac{1}{3} \sum_{j \in N^{+}(i)} w_{i j} \sum_{p \in N^{-}(i)} w_{p i}$.

Case 3b: $\quad|\{i, j\} \cap\{p, q\}|=1$ and $j=p$. Similarly to Case 3a, we obtain $\sum_{i j, j q \in A} \mathbb{E}\left(\epsilon_{i j} \epsilon_{j q}\right)=-\frac{1}{3} \sum_{i \in N^{-}(j)} w_{i j} \sum_{q \in N^{+}(j)} w_{j q}$.

Equation (11) and the subsequent computations imply that $4 \cdot \mathbb{E}\left(X^{2}\right)=W^{(2)}+$ $\frac{1}{3}(Q-R)$, where

$$
Q=\sum_{i \in V}\left(\left(\sum_{j \in N^{+}(i)} w_{i j}\right)^{2}-\sum_{j \in N^{+}(i)} w_{i j}^{2}+\left(\sum_{j \in N^{-}(i)} w_{j i}\right)^{2}-\sum_{j \in N^{-}(i)} w_{j i}^{2}\right),
$$

and

$$
R=2 \cdot \sum_{i \in V}\left(\sum_{j \in N^{+}(i)} w_{i j}\right)\left(\sum_{j \in N^{-}(i)} w_{j i}\right) .
$$

By the inequality of arithmetic and geometric means, for each $i \in V$, we have

$$
\left(\sum_{j \in N^{+}(i)} w_{i j}\right)^{2}+\left(\sum_{j \in N^{-}(i)} w_{j i}\right)^{2}-2\left(\sum_{j \in N^{+}(i)} w_{i j}\right)\left(\sum_{j \in N^{-}(i)} w_{j i}\right) \geq 0 .
$$

Therefore,

$$
Q-R \geq-\sum_{i \in V} \sum_{j \in N^{+}(i)} w_{i j}^{2}-\sum_{i \in V} \sum_{j \in N^{-}(i)} w_{j i}^{2}=-2 W^{(2)},
$$

and $4 \cdot \mathbb{E}\left(X^{2}\right) \geq W^{(2)}-2 W^{(2)} / 3=W^{(2)} / 3$, implying $\mathbb{E}\left(X^{2}\right) \geq W^{(2)} / 12$.

Now we can prove the main result of this section.

Theorem 1. The problem LOALB admits a kernel with $O\left(k^{2}\right)$ arcs.

Proof. Let $H$ be a digraph. We know that the answer to LOALB for $H$ is YES if and only if the answer to LOALB is YES for a digraph $D$ obtained from $H$ using Reduction Rule 1 as long as possible. Observe that $D$ is an oriented graph. Let $\mathcal{B}$ be the set of bijections from $V$ to $\{1, \ldots, n\}$. Observe that $f: \mathcal{B} \rightarrow \mathcal{B}$ such that $f(\alpha(v))=|V|+1-\alpha(v)$ for each $\alpha \in \mathcal{B}$ is a bijection. Note that $X(f(\alpha))=-X(\alpha)$ for each $\alpha \in \mathcal{B}$. Therefore, $\operatorname{Prob}(X=a)=\operatorname{Prob}(X=-a)$ for each real $a$ and, thus, $X$ is symmetric. Thus, by Lemmas 1 and 4 , we have $\operatorname{Prob}\left(X \geq \sqrt{W^{(2)} / 12}\right)>0$. Hence, if $\sqrt{W^{(2)} / 12} \geq k$, there is a bijection $\alpha: V \rightarrow\{1, \ldots, n\}$ such that $X(\alpha) \geq k$ and, thus, the answer to LOALB (for both $D$ and $H$ ) is Yes. Otherwise, $|A| \leq W^{(2)}<12 \cdot k^{2}$.

We close this section by outlining how Theorem 1 can be used to actually find a solution to LOALB if one exists. Let $(D, k)$ be an instance of LOALB where $D=(V, A)$ is a directed graph with integral positive arc-weights and $k \geq 1$ is an integer. Let $W$ be the total weight of $D$. As discussed above, we may assume 
that $D$ is an oriented graph. If $|A|<12 k^{2}$ then we can find a solution, if one exists, by trying all subsets $A^{\prime} \subseteq A$, and testing whether $\left(V, A^{\prime}\right)$ is acyclic and has weight at least $W / 2+k$; this search can be carried out in time $2^{O\left(k^{2}\right)}$. Next we assume $|A| \geq 12 k^{2}$. We know by Theorem 1 that $(D, k)$ is a YES-instance; it remains to find a solution.

For a vertex $i \in V$ let $d_{D}(i)$ denote its unweighted degree in $D$, i.e., the number of arcs (incoming or outgoing) that are incident with $i$. Consider the following reduction rule:

Reduction Rule 2. If there is a vertex $i \in V$ with $|A|-12 k^{2} \geq d_{D}(i)$, then delete $i$ from $D$.

Observe that by applying the rule we obtain again a YES-instance $(D-i, k)$ of LOALB since $D-i$ has still at least $12 k^{2}$ arcs. Moreover, if we know a solution $D_{i}^{\prime}$ of $(D-i, k)$, then we can efficiently obtain a solution $D^{\prime}$ of $(D, k)$ : if $\sum_{j \in N^{+}(i)} w_{i j} \geq \sum_{j \in N^{-}(i)} w_{i j}$ then we add $i$ and all outgoing arcs $i j \in A$ to $D_{i}^{\prime}$; otherwise, we add $i$ and all incoming $\operatorname{arcs} j i \in A$ to $D_{i}^{\prime}$. After multiple applications of Rule 2 we are left with an instance $\left(D_{0}, k\right)$ to which Rule 2 cannot be applied. Let $D_{0}=\left(V_{0}, A_{0}\right)$. We pick a vertex $i \in V_{0}$. If $i$ has a neighbor $j$ with $d_{D_{0}}(j)=1$, then $\left|A_{0}\right| \leq 12 k^{2}$, since $\left|A_{0}\right|-d_{D_{0}}(j)<12 k^{2}$. On the other hand, if $d_{D_{0}}(j) \geq 2$ for all neighbors $j$ of $i$, then $i$ has less than $2 \cdot 12 k^{2}$ neighbors, since $D_{0}-i$ has less than $12 k^{2}$ arcs; thus $\left|A_{0}\right|<3 \cdot 12 k^{2}$. Therefore, as above, time $2^{O\left(k^{2}\right)}$ is sufficient to try all subsets $A_{0}^{\prime} \subseteq A_{0}$ to find a solution to the instance $\left(D_{0}, k\right)$. Let $n$ denote the input size of instance $(D, k)$. Rule 2 can certainly be applied in polynomial time $n^{O(1)}$, and we apply it less than $n$ times. Hence, we can find a solution to $(D, k)$, if one exists, in time $n^{O(1)}+2^{O\left(k^{2}\right)}$.

Recall that a kernelization reduces in polynomial time an instance $(I, k)$ of a parameterized problem to a decision-equivalent instance $\left(I^{\prime}, k^{\prime}\right)$, its problem kernel, where $k^{\prime} \leq k$ and the size of $I^{\prime}$ is bounded by a function of $k$. Solutions for $(I, k)$ and solutions for $\left(I^{\prime}, k^{\prime}\right)$ are possibly unrelated to each other. We call $\left(I^{\prime}, k^{\prime}\right)$ a faithful problem kernel if from a solution for $\left(I^{\prime}, k^{\prime}\right)$ we can construct a solution for $(I, k)$ in time polynomial in $|I|$ and $k$. Clearly the above $\left(D_{0}, k\right)$ is a faithful kernel.

\section{Max Lin-2}

Consider a system of $m$ linear equations $e_{1}, \ldots, e_{m}$ in $n$ variables $z_{1}, \ldots, z_{n}$ over $\mathrm{GF}(2)$, and suppose that each equation $e_{j}$ has a positive integral weight $w_{j}$, $j=1, \ldots, m$. The problem MAX Lin-2 asks for an assignment of values to the variables that maximizes the total weight of the satisfied equations. Let $W=w_{1}+\cdots+w_{m}$.

To see that the total weight of the equations that can be satisfied is at least $W / 2$, we describe a simple procedure suggested in [13. We assign values to the variables $z_{1}, \ldots, z_{n}$ one by one and simplify the system after each assignment. When we wish to assign 0 or 1 to $z_{i}$, we consider all equations reduced to the form $z_{i}=b$, for a constant $b$. Let $W^{\prime}$ be the total weight of all such equations. 
We set $z_{i}:=0$, if the total weight of such equations is at least $W^{\prime} / 2$, and set $z_{i}:=1$, otherwise. If there are no equations of the form $z_{i}=b$, we set $z_{i}:=0$. To see that the lower bound $W / 2$ is tight, consider a system consisting of pairs of equations of the form $\sum_{i \in I} z_{i}=1$ and $\sum_{i \in I} z_{i}=0$ where both equations have the same weight.

The parameterized complexity of MAx Lin-2 parameterized above the tight lower bound $W / 2$ was stated by Mahajan, Raman and Sikdar [16] as an open question:

\section{Max Lin-2 Parameterized Above Tight Lower Bound (LinAlB)}

Instance: A system $S$ of $m$ linear equations $e_{1}, \ldots, e_{m}$ in $n$ variables $z_{1}, \ldots, z_{n}$ over $\mathrm{GF}(2)$, each equation $e_{i}$ with a positive integral weight $w_{i}, i=1,2, \ldots, m$, and a positive integer $k$. Each equation $e_{j}$ can be written as $\sum_{i \in I_{j}} z_{i}=b_{j}$, where $\emptyset \neq I_{j} \subseteq\{1, \ldots, n\}$.

Parameter: The integer $k$.

Question: Is there an assignment of values to the variables $z_{1}, \ldots, z_{n}$ such that the total weight of the satisfied equations is at least $W / 2+$ $k$, where $W=\sum_{i=1}^{m} w_{i}$ ?

Let $r_{j}$ be the number of variables in equation $e_{j}$, and let $r(S)=\max _{i=1}^{m} r_{j}$. We are not able to determine whether LINALB is fixed-parameter tractable or not, but we can prove that the following three special cases are fixed-parameter tractable: (1) there is a set $U$ of variables such that each equation contains an odd number of variables from $U,(2)$ there is a constant $r$ such that $r(S) \leq r$, (3) there is a constant $\rho$ such that any variable appears in at most $\rho$ equations.

Notice that in our formulation of LINALB it is required that each equation has a positive integral weight. In a relaxed setting in which an equation may have any positive real number as its weight, the problem is NP-complete even for $k=1$ and each $r_{j}=2$. Indeed, let each linear equation be of the form $z_{u}+z_{v}=1$. Then the problem is equivalent to MAXCUT, the problem of finding a cut of total weight at least $L$ in an undirected graph $G$, where $V(G)$ is the set of variables, $E(G)$ contains $\left(z_{u}, z_{v}\right)$ if and only if there is a linear equation $z_{u}+z_{v}=1$, and the weight of an edge $\left(z_{u}, z_{v}\right)$ equals the weight of the corresponding linear equation. The problem MAXCuT is a well-known NPcomplete problem. Let us transform an instance $I$ of MAXCuT into an instance $I^{\prime}$ of the "relaxed" LinALB by replacing the weight $w_{i}$ by $w_{i}^{\prime}:=w_{i} /(L-W / 2)$. We may assume that $L-W / 2>0$ since otherwise the instance is immediately seen as a YES-instance. Observe that the new instance $I^{\prime}$ has an assignment of values with total weight at least $W^{\prime} / 2+1$ if and only if $I$ has a cut with total weight at least $L$. We are done.

Let $A$ be the matrix of the coefficients of the variables in $S$. It is well-known that the maximum number of linearly independent columns of $A$ equals $\operatorname{rank} A$, and such a collection of columns can be found in time polynomial in $n$ and $m$, using, e.g., the Gaussian elimination on columns [3]. We have the following reduction rule and supporting lemma. 
Reduction Rule 3. Let $A$ be the matrix of the coefficients of the variables in $S$, let $t=\operatorname{rank} A$ and let columns $a^{i_{1}}, \ldots, a^{i_{t}}$ of $A$ be linearly independent. Then delete all variables not in $\left\{z_{i_{1}}, \ldots, z_{i_{t}}\right\}$ from the equations of $S$.

Lemma 5. Let $T$ be obtained from $S$ by Rule 3. Then $T$ is a YeS-instance if and only if $S$ is a YES-instance. Moreover, $T$ can be obtained from $S$ in time polynomial in $n$ and $m$.

Proof. If $t=n$, set $T:=S$, so assume that $t<n$. The remark before the lemma immediately implies that $T$ can be obtained from $S$ in time polynomial in $n$ and $m$. Let $S^{\prime}$ be a system of equations from $S$ and let $T^{\prime}$ be the corresponding system of equations from $T$. It is sufficient to prove the following claim:

There is an assignment of values to $z_{1}, \ldots, z_{n}$ satisfying all equations in $S^{\prime}$ and falsifying the rest of equations in $S$ if and only if there is an assignment of values to $z_{i_{1}}, \ldots, z_{i_{t}}$ satisfying all equations in $T^{\prime}$ and falsifying the rest of equations in $T$.

Let an assignment $z^{0}$ of values to $z=\left(z_{1}, \ldots, z_{n}\right)$ satisfy all equations of $S^{\prime}$ and falsify the equations of $S^{\prime \prime}$, where $S^{\prime \prime}=S \backslash S^{\prime}$. This assignment satisfies all equations of $R$, the system obtained from $S$ by replacing the right hand side $b_{j}$ of each equation in $S^{\prime \prime}$ by $1-b_{j}$. Note that $R$ has the same matrix $A$ of coefficients with columns $a^{1}, \ldots, a^{n}$. Let a column $a^{i} \notin\left\{a^{i_{1}}, \ldots, a^{i_{t}}\right\}$. Then, by definition of $a^{i_{1}}, \ldots, a^{i_{t}}, a^{i}=\lambda_{1} a^{i_{1}}+\cdots+\lambda_{t} a^{i_{t}}$ for some numbers $\lambda_{j} \in\{0,1\}$. Knowing the numbers $\lambda_{j}$, we may eliminate a variable $z_{i}$ from $R$ by replacing $a^{i}$ with the sum of all columns from $\left\{a^{i_{1}}, \ldots, a^{i_{t}}\right\}$ for which $\lambda_{j}=1$ and carrying out the obvious simplification of the system. Thus, we may eliminate from $R$ all variables $z_{i} \notin\left\{z_{i_{1}}, \ldots, z_{i_{t}}\right\}$ and get $y_{i_{1}} a^{i_{1}}+\cdots+y_{i_{t}} a^{i_{t}}=b^{\prime}$, where $b^{\prime}$ is the right hand side of $R$ and each $y_{j} \in\{0,1\}$. Now replace, in the modified $R$, the right hand side $b_{j}^{\prime}$ of each equation corresponding to an equation in $S^{\prime \prime}$ by $1-b_{j}^{\prime}$ obtaining $T$. Clearly, $\left(y_{i_{1}}, \ldots, y_{i_{t}}\right)$ satisfies all equations of $T^{\prime}$ and falsifies all equations in $T^{\prime \prime}=T \backslash T^{\prime}$.

Suppose now that $\left(y_{i_{1}}, \ldots, y_{i_{t}}\right)$ satisfies all equations of $T^{\prime}$ and falsifies all equations in $T^{\prime \prime}$. Then $\left(y_{1}, \ldots, y_{n}\right)$, where $y_{j}=0$ if $j \notin\left\{i_{1}, \ldots, i_{t}\right\}$, satisfies all equations of $S^{\prime}$ and falsifies all equations in $S^{\prime \prime}$. Thus, the claim has been proved.

Consider the following reduction rule for LinALB used in [13].

Reduction Rule 4. If we have, for a subset I of $\{1,2, \ldots, n\}$, the equation $\sum_{i \in I} z_{i}=b^{\prime}$ with weight $w^{\prime}$, and the equation $\sum_{i \in I} z_{i}=b^{\prime \prime}$ with weight $w^{\prime \prime}$, then we replace this pair by one of these equations with weight $w^{\prime}+w^{\prime \prime}$ if $b^{\prime}=b^{\prime \prime}$ and, otherwise, by the equation whose weight is bigger, modifying its new weight to be the difference of the two old ones. If the resulting weight is 0 , we omit the equation from the system.

If Rule 4 is not applicable to a system we call the system reduced under Rule 4. Note that the problem LiNALB for $S$ and the system obtained from $S$ by applying Rule 4 as long as possible have the same answer. 
Let $I_{j} \subseteq\{1, \ldots, n\}$ be the set of indices of the variables participating in equation $e_{j}$, and let $b_{j} \in\{0,1\}$ be the right hand side of $e_{j}$. Define a random variable $X=\sum_{j=1}^{m} X_{j}$, where $X_{j}=(-1)^{b_{j}} w_{j} \prod_{i \in I_{j}} \epsilon_{i}$ and all the $\epsilon_{i}$ are independent uniform random variables on $\{-1,1\}$ ( $X$ was first introduced in [13]). We set $z_{i}=0$ if $\epsilon_{i}=1$ and $z_{i}=1$, otherwise, for each $i$. Observe that $X_{j}=w_{j}$ if $e_{j}$ is satisfied and $X_{j}=-w_{j}$, otherwise.

Lemma 6. Let $S$ be reduced under Rule 4. The weight of the satisfied equations is at least $W / 2+k$ if and only if $X \geq 2 k$. We have $\mathbb{E}(X)=0$ and $\mathbb{E}\left(X^{2}\right)=$ $\sum_{j=1}^{m} w_{j}^{2}$.

Proof. Observe that $X$ is the difference between the weights of satisfied and non-satisfied equations. Therefore, the weight of the satisfied equations equals $(X+W) / 2$, and it is at least $W / 2+k$ if and only if $X \geq 2 k$. Since $\epsilon_{i}$ are independent, $\mathbb{E}\left(\prod_{i \in I_{j}} \epsilon_{i}\right)=\prod_{i \in I_{j}} \mathbb{E}\left(\epsilon_{i}\right)=0$. Thus, $\mathbb{E}\left(X_{j}\right)=0$ and $\mathbb{E}(X)=0$ by linearity of expectation. Moreover,

$$
\mathbb{E}\left(X^{2}\right)=\sum_{j=1}^{m} \mathbb{E}\left(X_{j}^{2}\right)+\sum_{1 \leq j \neq q \leq m} \mathbb{E}\left(X_{j} X_{q}\right)=\sum_{j=1}^{m} w_{j}^{2}>0
$$

as $\mathbb{E}\left(\prod_{i \in I_{j}} \epsilon_{i} \cdot \prod_{i \in I_{q}} \epsilon_{i}\right)=\mathbb{E}\left(\prod_{i \in I_{j} \Delta I_{q}} \epsilon_{i}\right)=0$ implies $\mathbb{E}\left(X_{j} X_{q}\right)=0$, where $I_{j} \Delta I_{q}$ is the symmetric difference between $I_{j}$ and $I_{q}\left(I_{j} \Delta I_{q} \neq \emptyset\right.$ due to Reduction Rule (4).

Lemma 7. Let $S$ be reduced under Rule 4 and suppose that no variable appears in more than $\rho \geq 2$ equations of $S$. Then $\mathbb{E}\left(X^{4}\right) \leq 2 \rho^{2}\left(\mathbb{E}\left(X^{2}\right)\right)^{2}$.

Proof. Observe that

$$
\mathbb{E}\left(X^{4}\right)=\sum_{(p, q, s, t) \in[m]^{4}} \mathbb{E}\left(X_{p} X_{q} X_{s} X_{t}\right),
$$

where $[m]=\{1, \ldots, m\}$. Note that if the product $X_{p} X_{q} X_{s} X_{t}$ contains a variable $\epsilon_{i}$ in only one or three of the factors, then $\mathbb{E}\left(X_{p} X_{q} X_{s} X_{t}\right)=A \cdot \mathbb{E}\left(\epsilon_{i}\right)=0$, where $A$ is a polynomial in random variables $\epsilon_{l}, l \in\{1, \ldots, n\} \backslash\{i\}$. Thus, the only nonzero terms in (2) are those for which either (1) $p=q=s=t$, or (2) there are two distinct integers $j, l$ such that each of them coincides with two elements in the sequence $p, q, s, t$, or (3) $|\{p, q, s, t\}|=4$, but each variable $\epsilon_{i}$ appears in an even number of the factors in $X_{p} X_{q} X_{s} X_{t}$. In Cases 1 and 2, we have $\mathbb{E}\left(X_{p} X_{q} X_{s} X_{t}\right)=w_{p}^{4}$ and $\mathbb{E}\left(X_{p} X_{q} X_{s} X_{t}\right)=w_{j}^{2} w_{l}^{2}$, respectively. In Case 3,

$$
\mathbb{E}\left(X_{p} X_{q} X_{s} X_{t}\right) \leq w_{p} w_{q} w_{s} w_{t} \leq\left(w_{p}^{2} w_{q}^{2}+w_{s}^{2} w_{t}^{2}\right) / 2 .
$$

Let $1 \leq j<l \leq m$. Observe that $\mathbb{E}\left(X_{p} X_{q} X_{s} X_{t}\right)=w_{j}^{2} w_{l}^{2}$ in Case 2 for $\left(\begin{array}{l}4 \\ 2\end{array}\right)=64$-tuples $(p, q, s, t) \in[m]^{4}$. In Case 3, we claim that $j, l \in\{p, q, s, t\}$ for at most $4 \cdot(\rho-1)^{2} 4$-tuples $(p, q, s, t) \in[m]^{4}$. To see this, first note that $w_{p}^{2} w_{q}^{2}$ and $w_{s}^{2} w_{t}^{2}$ appear in our upper bound on $\mathbb{E}\left(X_{p} X_{q} X_{s} X_{t}\right)$ (with coefficient $\left.1 / 2\right)$. 
Therefore, there are only four possible ways for $w_{j}^{2} w_{l}^{2}$ to appear in our upper bound, namely the following: (i) $j=p, l=q$, (ii) $l=p, j=q$, (iii) $j=s, l=t$, and (iv) $l=s, j=t$. Now assume, without loss of generality, that $j=p$ and $l=q$. Since $S$ is reduced under Rule 4 , the product $X_{j} X_{l}$ must have a variable $\epsilon_{i}$ of degree one. Thus, $\epsilon_{i}$ must be in $X_{s}$ or $X_{t}$, but not in both (two choices). Assume that $\epsilon_{i}$ is in $X_{s}$. Observe that there are at most $\rho-1$ choices for $s$. Note that $X_{j} X_{l} X_{s}$ must contain contain a variable $\epsilon_{i^{\prime}}$ of odd degree. Thus, $\epsilon_{i^{\prime}}$ must be in $X_{t}$ and, hence, there are at most $\rho-1$ choices for $t$.

Therefore, we have

$$
\mathbb{E}\left(X^{4}\right) \leq \sum_{j=1}^{m} w_{j}^{4}+\left(6+4(\rho-1)^{2}\right) \sum_{1 \leq j<l \leq m} w_{j}^{2} w_{l}^{2}<2 \rho^{2}\left(\sum_{j=1}^{m} w_{j}^{2}\right)^{2} .
$$

Thus, by Lemma $6 \mathbb{E}\left(X^{4}\right) \leq 2 \rho^{2}\left(\mathbb{E}\left(X^{2}\right)\right)^{2}$.

Case 1 of Theorem 2 is of interest since its condition can be checked in polynomial time due to the following:

Proposition 1. We can check, in polynomial time, whether there exists a set $U$ of variables such that each equation of $S$ contains an odd number of variables from $U$.

Proof. Observe that such a set $U$ exists if and only if the unweighted system $S^{\prime}$ of linear equations over $\mathrm{GF}(2)$ obtained from $S$ by replacing each $b_{j}$ with 1 has a solution. Indeed, if $U$ exists, set $z_{j}=1$ for each $z_{j} \in U$ and $z_{j}=0$ for each $z_{j} \notin U$. This assignment is a solution to $S^{\prime}$. If a solution to $S^{\prime}$ exists, form $U$ by including in it all variables $z_{j}$ which equal 1 in the solution. We can check whether $S^{\prime}$ has a solution using the Gaussian elimination or other polynomial-time algorithms, see, e.g., [5].

Now we can prove the following:

Theorem 2. Let $S$ be reduced under Rule 4. The following three special cases of LINALB are fixed-parameter tractable: (1) there is a set $U$ of variables such that each equation contains an odd number of variables from $U$, (2) there is a constant $r$ such that $r(S) \leq r$, (3) there is a constant $\rho$, such that any variable appears in at most $\rho$ equations. In each case, there exists a kernel with $O\left(k^{2}\right)$ equations and variables.

Proof. Case 1. Let $z^{0}=\left(z_{1}^{0}, \ldots, z_{n}^{0}\right) \in\{0,1\}^{n}$ be an assignment of values to the variables $z_{1}, \ldots, z_{n}$, and let $-z^{0}=\left(z_{1}^{\prime}, \ldots, z_{n}^{\prime}\right)$, where $z_{i}^{\prime}=1-z_{i}^{0}$ if $z_{i} \in U$ and $z_{i}^{\prime}=z_{i}^{0}$, otherwise, $i=1, \ldots, n$. Observe that $f: z^{0} \mapsto-z^{0}$ is a bijection on the set of assignments and $X\left(-z^{0}\right)=-X\left(z^{0}\right)$. Thus, $X$ is a symmetric random variable. Therefore, by Lemmas 1 and 6 , Prob $(X \geq \sqrt{m}) \geq$ $\operatorname{Prob}\left(X \geq \sqrt{\sum_{j=1}^{m} w_{j}^{2}}\right)>0$. Hence, if $\sqrt{m} \geq 2 k$, the answer to LinALB is YES. Otherwise, $m<4 k^{2}$ and after applying Rule 3, we obtain a kernel with $O\left(k^{2}\right)$ equations and variables. 
Case 2. Since $X$ is a polynomial of degree at most $r$, it follows by Lemma 3 that $\mathbb{E}\left(X^{4}\right) \leq 2^{6 r} \mathbb{E}\left(X^{2}\right)^{2}$. This inequality and the results in the previous paragraph show that the conditions of Lemma 2 are satisfied and, thus,

$$
\operatorname{Prob}\left(X>\frac{\sqrt{\sum_{j=1}^{m} w_{j}^{2}}}{4 \cdot 8^{r}}\right)>0, \quad \text { implying } \operatorname{Prob}\left(X>\frac{\sqrt{m}}{4 \cdot 8^{r}}\right)>0 .
$$

Consequently, if $2 k-1 \leq \sqrt{m} /\left(4 \cdot 8^{r}\right)$, then there is an assignment of values to the variables $z_{1}, \ldots, z_{n}$ which satisfies equations of total weight at least $W / 2+k$. Otherwise, $2 k-1>\sqrt{m} /\left(4 \cdot 8^{r}\right)$ and $m<16(2 k-1)^{2} 64^{r}$. After applying Rule 3. we obtain the required kernel.

Case 3. If $\rho=1$, it is easy to find an assignment to the variables that satisfies all equations of $S$. Thus, we may assume that $\rho \geq 2$. To prove that there exists a kernel with $O\left(k^{2}\right)$ equations, we can proceed as in Case 2, but use Lemma 7 rather than Lemma 3 .

Remark 1. Note that even if $S$ does not satisfy Case 2 of the theorem, $T$, the system obtained from $S$ using Rule 3, may still satisfy Case 2. However, we have not formulated the theorem for $S$ reduced under Rule 3 as the reduced system depends on the choice of a maximum linear independent collection of columns of $A$.

Remark 2. Unfortunately, we cannot use Lemma 2 for $X$ to prove that the whole problem LinALB is fixed-parameter tractable. This is due to the fact that $\mathbb{E}\left(X^{4}\right) \geq \Theta\left(\mathbb{E}\left(X^{2}\right)^{3}\right)$ for some systems $S$. One such system is $\sum_{i \in I} z_{i}=1$ for each nonempty subset $I$ of $\{1, \ldots, n\}$. (Thus, $m=2^{n}-1$.) We set the weight $w_{j}=1$ for every $j=1, \ldots, m$. By Lemma $6, \mathbb{E}\left(X^{2}\right)=m$. Let $Q_{n}$ be the set of 4-tuples $(p, q, s, t) \in[m]^{4}$, where $[m]=\{1, \ldots, m\}$, such that $|\{p, q, s, t\}|=4$ and each variable $\epsilon_{i}$ appears in an even number of the factors in $X_{p} X_{q} X_{s} X_{t}$. By the proof of Lemma 7 we have $\mathbb{E}\left(X^{4}\right)>\sum_{(p, q, s, t) \in Q} X_{p} X_{q} X_{s} X_{t}=\left|Q_{n}\right|$. Let $(p, q, s, t) \in Q_{n-1}$ (we allow only variables $\epsilon_{1}, \ldots, \epsilon_{n-1}$ in $X_{p}, X_{q}, X_{s}$ and $\left.X_{t}\right)$. We can construct eight 4 -tuples in $Q_{n}$ using $(p, q, s, t)$ as a starting point. Indeed, we can multiply each of $X_{p}, X_{q}, X_{s}$ and $X_{t}$ by $\epsilon_{n}$, or exactly two of $X_{p}, X_{q}, X_{s}$ and $X_{t}$ by $\epsilon_{n}$, or keep $X_{p}, X_{q}, X_{s}$ and $X_{t}$ without a change. Thus, $\left|Q_{n}\right| \geq 8\left|Q_{n-1}\right|$. Therefore, $\mathbb{E}\left(X^{4}\right)>\left|Q_{n-3}\right| \geq 8^{n-3}>m^{3} / 8^{3}=\mathbb{E}\left(X^{2}\right)^{3} / 8^{3}$ as $\left|Q_{3}\right| \geq 1$.

A faithful kernel can be found for all three cases of Theorem2, but we restrict ourselves to Case 2. Consider an instance $(S, k)$ of LINALB over $n$ variables with $r=r(S)=O(1),|S|=m \geq f(k, r)$, where $f(k, r)=16(2 k-1)^{2} 64^{r}$ and assume $S$ is reduced under Reduction Rule 4 . We can find a solution for $(S, k)$ (i.e., an assignment satisfying at least $W / 2+k$ equations) in polynomial time by using the observation from [1] that the random variables $\epsilon_{i}$ are $4 r$-wise independent and, thus, one can use an $O\left(n^{2 r}\right)$-size sample space to support each $\epsilon_{i}$ (for more details and the sample space construction, see [1]). 
Alternatively, we can use a modification of the approach given earlier for LOALB to obtain in polynomial time a faithful kernel. Similar approaches can be used to obtain faithful kernels for Cases 1 and 3. Consider the following reduction rule:

Reduction Rule 5. If there is some variable $x$ of $S$ that occurs in at most $|S|-f(k, r)$ equations, then remove all the equations from $S$ in which $x$ occurs.

Let $T$ be the system obtained from $S$ using Rule 3 . Apply Rule 5 to $T$ as long as possible. Note that we can transform a solution for $(T, k)$ to a solution for $(S, k)$ by assigning zero to all variables not in $T$. To show that the number of equations in $T$ is polynomially bounded in $k$, let $x$ be a variable of $T$ that occurs in the smallest number of equations of $T$, and let $T=T_{1} \cup T_{2}$ where all equations in $T_{1}$ contain $x$ and no equation in $T_{2}$ contains $x$. Since $T$ is reduced under Rule 5. $\left|T_{2}\right|<f(k, r)=O\left(k^{2}\right)$; thus $T_{2}$ involves at most $r \cdot f(k, r)=O\left(k^{2}\right)$ variables. However, by Rule 3 and the choice of $x, x$ is the only variable that occurs in $T_{1}$ but does not occur in $T_{2}$. Hence $\left|T_{1}\right|=O\left((r \cdot f(k, r))^{r-1}\right)=O\left(k^{2 r-2}\right)$ and $|T|=O\left(k^{2}+k^{2 r-2}\right)$.

\section{Max Exact $r$-SAT}

Let $x=\left(x_{1}, x_{2}, \ldots, x_{n}\right)$ be a vector of Boolean variables. We assume that each variable $x_{i}$ attains 1 or -1 (meaning TRUE and FALSE, respectively). We will denote the negation of a variable $x_{i}$ by $-x_{i}$ and we let $L=\left\{x_{1}, x_{2}, \ldots\right.$, $\left.x_{n},-x_{1},-x_{2}, \ldots,-x_{n}\right\}$ be the set of literals over $x_{1}, \ldots, x_{n}$. Let $r \geq 2$ be a fixed integer, and let $C=\left\{C_{1}, C_{2}, \ldots, C_{m}\right\}$ be a set of clauses, each involving exactly $r$ distinct literals from $L$ such that for no pair $y, z$ of literals we have $y=-z$. Then $\mathcal{C}$ is an exact $r-C N F$ formula.

Consider an exact $r$-CNF formula $\mathcal{C}$ with $m$ clauses and a random truth assignment for $x$. Since the probability of a clause of $\mathcal{C}$ to be satisfied is $1-2^{-r}$, the expected number of satisfied clauses in $\mathcal{C}$ is $\left(1-2^{-r}\right) m$. Thus, there is a truth assignment for $x$ that satisfies at least $\left(1-2^{-r}\right) m$ clauses. This bound is tight as can be seen by considering an $r$-CNF formula that contains all $2^{r}$ possible clauses over the same $r$ variables, or by considering a disjoint union of several such $r$-CNF formulas.

Mahajan, Raman and Sikdar [16] stated the complexity of the following problem as an open question.

\section{ExACt $r$-SAT Above Tight Lower Bound ( $r$-SATALB)}

Instance: An exact $r$-CNF formula $\mathcal{C}$ and a positive rational number $k$ with denominator $2^{r}$.

Parameter: The number $k$.

Question: Is there a truth assignment $\left(x_{1}^{\prime}, x_{2}^{\prime}, \ldots, x_{n}^{\prime}\right)$ satisfying at least $\left(1-2^{-r}\right) m+k$ clauses of $\mathcal{C}$ ? 
Mahajan, Raman and Sikdar [16] require $k$ to be a positive integer, but since $\left(1-2^{-r}\right) m$ is a positive rational number with denominator $2^{r}$, our setting for $k$ seems more natural.

We will prove that the problem has a quadratic kernel for a wide family of instances. We say that a pair of distinct clauses $Y$ and $Z$ has a conflict if there is a literal $p \in Y$ such that $-p \in Z$. We say that a pair $Y$ and $Z$ has an overlap if $Y$ and $Z$ have common literals, but they do not have a conflict. For an exact $r$-CNF formula $\mathcal{C}$, the conflict number $\operatorname{cn}(\mathcal{C})=c-o$, where $c$ $(o$, respectively) is the number of ordered pairs of clauses having a conflict (an overlap, respectively).

Theorem 3. The problem $r$-SATALB restricted to exact $r$-CNF formulas with $m$ clauses and of conflict number at most $\left(2^{r}-2\right) m$ admits a quadratic kernel.

To establish the theorem, consider an exact $r$-CNF formula $\mathcal{C}$ with $m$ clauses and of conflict number at most $\left(2^{r}-2\right) m$, and consider a clause $Z$ of $\mathcal{C}$. Let $x_{i_{1}}, x_{i_{2}}, \ldots, x_{i_{r}}$ be the variables corresponding to the literals of $Z$ and let $x_{i_{1}}^{0}, x_{i_{2}}^{0}, \ldots, x_{i_{r}}^{0}$ be the unique truth assignment not satisfying $Z$. Define a random variable $X_{Z}$ as follows: Let $V=\{-1,1\}^{r}-\left\{\left(x_{i_{1}}^{0}, x_{i_{2}}^{0}, \ldots, x_{i_{r}}^{0}\right)\right\}$ and

$$
X_{Z}\left(x_{1}, x_{2}, \ldots, x_{n}\right)=-\frac{2^{r}-1}{2^{r}}+\sum_{\left(v_{1}, \ldots, v_{r}\right) \in V} \frac{\prod_{j=1}^{r}\left(1+x_{i_{j}} v_{j}\right)}{2^{r}} .
$$

The value of $X_{Z}$ at a truth assignment $x^{\prime}=\left(x_{1}^{\prime}, x_{2}^{\prime}, \ldots, x_{n}^{\prime}\right)$ is $2^{-r}$ if $x^{\prime}$ satisfies $Z$ (in this case the product equals $2^{r}$ ), and it is $2^{-r}-1$, otherwise. Let $X=$ $\sum_{Z \in \mathcal{C}} X_{Z}(X$ is a special case of a random variable introduced in [1]).

We study some properties of $X$ in the following two lemmas.

Lemma 8. Let $x^{\prime}=\left(x_{1}^{\prime}, x_{2}^{\prime}, \ldots, x_{n}^{\prime}\right)$ be a truth assignment. Then the value of $X$ at $x^{\prime}$ equals $m\left(x^{\prime}\right)-\left(1-2^{-r}\right) m$, where $m\left(x^{\prime}\right)$ is the number of clauses in $\mathcal{C}$ satisfied by $x^{\prime}$. Thus, the answer to $r$-SATALB is YES if and only if $X\left(x^{\prime \prime}\right) \geq k$ for some truth assignment $x^{\prime \prime}$. We also have $\mathbb{E}(X)=0$ and $\mathbb{E}\left(X^{4}\right) \leq 2^{6 r} \mathbb{E}\left(X^{2}\right)^{2}$.

Proof. Let $x^{\prime}=\left(x_{1}^{\prime}, x_{2}^{\prime}, \ldots, x_{n}^{\prime}\right)$ be a truth assignment. Observe that $X\left(x^{\prime}\right)=$ $m\left(x^{\prime}\right) 2^{-r}+\left(2^{-r}-1\right)\left(m-m\left(x^{\prime}\right)\right)=m\left(x^{\prime}\right)-\left(1-2^{-r}\right) m$. Hence, $m\left(x^{\prime}\right) \geq$ $\left(1-2^{-r}\right) m+k$ if and only if $X\left(x^{\prime}\right) \geq k$.

Observe that the probability of $Z$ being satisfied (not satisfied) is $1-2^{-r}$ $\left(2^{-r}\right)$. Thus, the expectation of $X_{Z}$ is zero, and $\mathbb{E}(X)=0$ by linearity of expectation. Since $X$ is a polynomial of degree at most $r$ in $x_{1}, x_{2}, \ldots, x_{n}$, it follows, by Lemma 3, that $\mathbb{E}\left(X^{4}\right) \leq 2^{6 r} \mathbb{E}\left(X^{2}\right)^{2}$.

Lemma 9. We have $\mathbb{E}\left(X^{2}\right) \geq m 4^{-r}$.

Proof. Observe that $\mathbb{E}\left(X^{2}\right)=\sum_{Z \in \mathcal{C}} \mathbb{E}\left(X_{Z}^{2}\right)+\sum_{Y \neq Z \in \mathcal{C}} \mathbb{E}\left(X_{Y} X_{Z}\right)$. We will compute $\mathbb{E}\left(X_{Z}^{2}\right)$ and $\mathbb{E}\left(X_{Y} X_{Z}\right)$ separately.

By the proof of Lemma $8, X_{Z}$ equals $2^{-r}$ with probability $1-2^{-r}$ and $2^{-r}-1$ with probability $2^{-r}$. Thus, $X_{Z}^{2}$ equals $2^{-2 r}$ with probability $1-2^{-r}$ and $\left(2^{-r}-1\right)^{2}$ with probability $2^{-r}$. Hence, $\mathbb{E}\left(X_{Z}^{2}\right)=2^{-r}-4^{-r}$. 
For a clause $Y$ of $\mathcal{C}$, let $\operatorname{vars}(Y)$ denote the sets of variables in $Y$ and let $\operatorname{lits}(Y)$ be the set of literals in $Y$. To evaluate $\mathbb{E}\left(X_{Y} X_{Z}\right)$, we consider the following three cases:

Case 1: $\quad \operatorname{vars}(Y) \cap \operatorname{vars}(Z)=\emptyset$. Then $X_{Y}$ and $X_{Z}$ are independent random variables and, thus, $\mathbb{E}\left(X_{Y} X_{Z}\right)=\mathbb{E}\left(X_{Y}\right) \mathbb{E}\left(X_{Z}\right)=0$.

Case 2: $\quad Y$ and $Z$ have a conflict. Then $X_{Y} X_{Z}$ equals $2^{-r}\left(2^{-r}-1\right)$ with probability $2^{-r+1}$, and $X_{Y} X_{Z}$ equals $2^{-2 r}$ with probability $1-2^{-r+1}$. Hence, $\mathbb{E}\left(X_{Y} X_{Z}\right)=-4^{-r}$.

Case 3: $|\operatorname{vars}(Y) \cap \operatorname{vars}(Z)|=t>0$ and $|\operatorname{lits}(Y) \cap \operatorname{lits}(Z)|=t$. Since $Y \neq Z$, we have $1 \leq t<r$. Without loss of generality, assume that $\operatorname{lits}(Y)=\left\{x_{1}, \ldots, x_{t}\right.$, $\left.x_{t+1}, \ldots x_{r}\right\}$ and $\operatorname{lits}(Z)=\left\{x_{1}, \ldots, x_{t}, x_{r+1}, \ldots x_{2 r-t}\right\}$. Thus, $X_{Y} X_{Z}$ equals $\left(2^{-r}-1\right)^{2}$ with probability $2^{t-2 r}, 2^{-r}\left(2^{-r}-1\right)$ with probability $\left(2^{r-t+1}-\right.$ $2) / 2^{2 r-t}$, and $2^{-2 r}$ with probability $\left(1-2^{r-t+1}+2^{2 r-t}\right) / 2^{2 r-t}$. Hence,

$$
\mathbb{E}\left(X_{Y} X_{Z}\right)=2^{t-2 r}\left(1-2^{-t}\right) \geq 4^{-r} .
$$

Since $\operatorname{cn}(\mathcal{C}) \leq\left(2^{r}-2\right) m$, we have

$\mathbb{E}\left(X^{2}\right) \geq \sum_{Z \in \mathcal{C}} \mathbb{E}\left(X_{Z}^{2}\right)+\sum_{Y \neq Z \in \mathcal{C}} \mathbb{E}\left(X_{Y} X_{Z}\right) \geq\left(2^{-r}-4^{-r}\right) m-\operatorname{cn}(\mathcal{C}) \cdot 4^{-r} \geq m 4^{-r}$.

Now we can complete the proof of Theorem 3 By Lemmas 2 , and 9 Prob $(X>$ $\left.\sqrt{m} /\left(2^{r} \cdot 4 \cdot 8^{r}\right)\right)>0$. Thus, if $\sqrt{m} /\left(2^{r} \cdot 4 \cdot 8^{r}\right) \geq k$, there is a truth assignment $x^{\prime}$ such that $X\left(x^{\prime}\right) \geq k$, i.e., the answer to the instance of $r$-SATALB is YES. Otherwise, $m<16 \cdot 256^{r} k^{2}$. Thus Theorem 3 is established.

Consider a YES-instance $(\mathcal{C}, k)$ of $r$-SATALB with $n$ variables, $m \geq 16 \cdot 256^{r} k^{2}$ clauses, and $\operatorname{cn}(\mathcal{C}) \leq\left(2^{r}-2\right) m$. As in the previous section, we can find in polynomial time a solution for $(\mathcal{C}, k)$ using the facts that each random variable $x_{i}$ is $4 r$-wise independent and there is an $O\left(n^{2 r}\right)$-size sample space to support each $x_{i}$.

\section{Discussions}

We have showed that the new method allows us to prove that some maximization problems parameterized above tight lower bounds are fixed-parameter tractable. Our method can also be used for minimization problems parameterized below tight upper bounds. As a simple example, consider the feedback arc problem: given a digraph $D=(V, A)$ find a minimum set $F$ of $\operatorname{arcs}$ such that $D-F$ is acyclic. Certainly, $|A| / 2$ is a tight upper bound on a minimum feedback set and we can consider the parameterized problem which asks whether $D$ has a 
feedback arc set with at most $|A| / 2-k$ arcs. Fixed-parameter tractability of this parameterized problem follows immediately from fixed-parameter tractability of LOALB, but we could prove this result directly using essentially the same approach as for LOALB.

It would be interesting to obtain applications of our method to other problems parameterized above tight lower bounds or below tight upper bounds. One such very recent application is given in [9], where an open problem due to Benny Chor and described in 17] was solved. The random variable $X$ considered there is not symmetric and both application of Lemma 3 and computation of $\mathbb{E}\left(X^{2}\right)$ are more involved than for the problems considered in this paper.

Let us provide further comments on the problems considered in this paper. First, it is natural to parameterize MAX LIN-2 not just by $k$ but also by $r=$ $r(S)$. The proof of Case 2 of Theorem 2 shows immediately that this twoparameter problem is fixed-parameter tractable (but our problem kernel is no longer of polynomial size). This result can be viewed as a contribution towards Multivariate Algorithmics as outlined by Fellows [7. Second, we have managed to obtain a polynomial problem kernel for 2-SATALB in [10. The approach there is very different from the method we introduced here and it involves signed weighted graphs, graph matching theory and the first moment probabilistic method. Perhaps, the approach of [10] can be extended to 3-SATALB, but we doubt that it can be extended to $r$-SATALB for $r>3$.

Acknowledgments. Research of Gutin, Kim and Yeo was supported in part by an EPSRC grant.

\section{References}

[1] N. Alon, G. Gutin, and M. Krivelevich. Algorithms with large domination ratio. J. Algorithms, 50(1):118-131, 2004.

[2] J. Bang-Jensen and G. Gutin. Digraphs. Springer Monographs in Mathematics. Springer-Verlag London Ltd., London, second edition, 2009.

[3] T. S. Blyth and E. F. Robertson, Basic Linear Algebra, Springer, 2000.

[4] J. Bourgain. Walsh subspaces of $L^{p}$-product spaces. In Seminar on Functional Analysis, 1979-1980 (French). École Polytech., Palaiseau, 1980. Exp. No. 4A, 9.

[5] D. Coppersmith. Solving linear systems over GF(2): block Lanczos algorithm. Lin. Algebra Applic., 192:33-60, 1993.

[6] R. G. Downey and M. R. Fellows. Parameterized Complexity. Monographs in Computer Science. Springer Verlag, 1999.

[7] M. R. Felows, Towards Fully Multivariate Algorithmics: Some New Results and Directions in Parameter Ecology. Proc. IWOCA 2009, Lect. Notes Comput. Sc., to appear. 
[8] J. Flum and M. Grohe. Parameterized Complexity Theory, volume XIV of Texts in Theoretical Computer Science. An EATCS Series. Springer Verlag, 2006.

[9] G. Gutin, E. J. Kim, M. Mnich, and A. Yeo. Ordinal Embedding Relaxations Parameterized Above Tight Lower Bound. Tech. Report arXiv:0907.5427, July 2009.

[10] G. Gutin, E. J. Kim, S. Szeider, and A. Yeo. Fixed-parameter tractability of Max 2-SAT parameteried above tight lower bound. Tech. Report arXiv:0907.4573, July 2009.

[11] G. Gutin, A. Rafiey, S. Szeider, and A. Yeo. The linear arrangement problem parameterized above guaranteed value. Theory Comput. Syst., 41:521538, 2007.

[12] G. Gutin, S. Szeider, and A. Yeo. Fixed-parameter complexity of minimum profile problems. Algorithmica, 52(2):133-152, 2008.

[13] J. Håstad and S. Venkatesh. On the advantage over a random assignment. In Proceedings of the Thirty-Fourth Annual ACM Symposium on Theory of Computing, pages 43-52, New York, 2002. ACM. Full version appeared in Random Structures Algorithms 25(2) (2004), pp. 117-149.

[14] P. Heggernes, C. Paul, J. A. Telle, and Y. Villanger. Interval completion with few edges. In STOC'O7-Proceedings of the 39th Annual ACM Symposium on Theory of Computing, pages 374-381. ACM, 2007. Full version appeared in SIAM J. Comput. 38(5), 2008/09.

[15] M. Mahajan and V. Raman. Parameterizing above guaranteed values: MaxSat and MaxCut. J. Algorithms, 31(2):335-354, 1999.

[16] M. Mahajan, V. Raman, and S. Sikdar. Parameterizing above or below guaranteed values. J. of Computer and System Sciences, 75(2):137-153, 2009 .

[17] R. Niedermeier. Invitation to Fixed-Parameter Algorithms. Oxford Lecture Series in Mathematics and its Applications. Oxford University Press, 2006.

[18] V. Vovk, Private communication, August, 2009. 